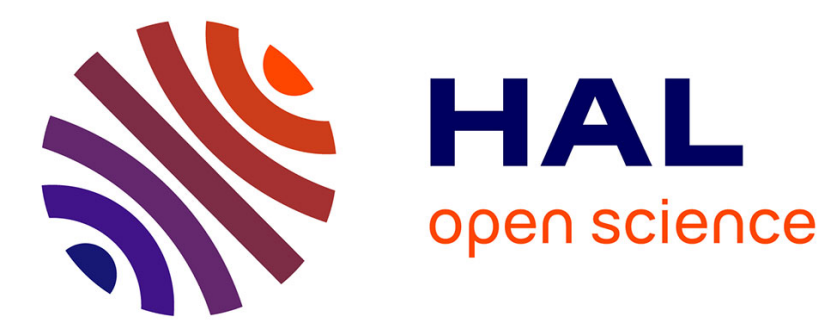

\title{
Toward design optimization of a Pelton turbine runner
}

Christian Vessaz, Loïc Andolfatto, François Avellan, Christophe Tournier

\section{To cite this version:}

Christian Vessaz, Loïc Andolfatto, François Avellan, Christophe Tournier. Toward design optimization of a Pelton turbine runner. Structural and Multidisciplinary Optimization, 2017, 10.1007/s00158-0161465-7. hal-01521737

\section{HAL Id: hal-01521737 \\ https://hal.science/hal-01521737}

Submitted on 12 May 2017

HAL is a multi-disciplinary open access archive for the deposit and dissemination of scientific research documents, whether they are published or not. The documents may come from teaching and research institutions in France or abroad, or from public or private research centers.
L'archive ouverte pluridisciplinaire HAL, est destinée au dépôt et à la diffusion de documents scientifiques de niveau recherche, publiés ou non, émanant des établissements d'enseignement et de recherche français ou étrangers, des laboratoires publics ou privés. 


\title{
Toward design optimization of a Pelton turbine runner
}

\author{
Christian Vessaz • Loïc \\ Andolfatto - François \\ Avellan · Christophe \\ Tournier
}
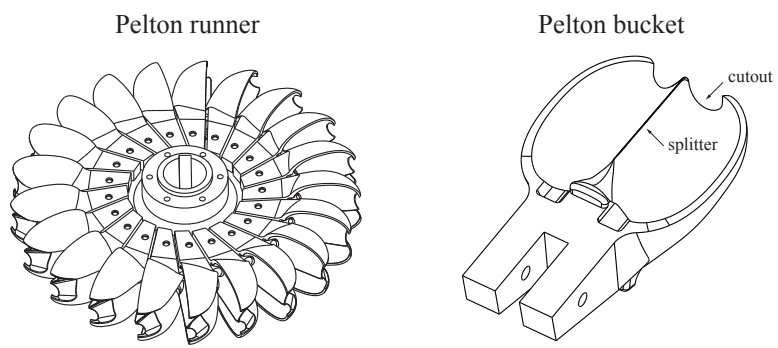

Fig. 1 Pelton runner and detail of a bucket.

gate model is built according to the simulated performance of these runners. Moreover, an original clustering approach is proposed to decompose the design problem into four sub-problems of smaller dimensions that can be addressed with more conventional optimization techniques.

\begin{abstract}
The objective of the present paper is to propose a strategy to optimize the performance of a Pelton runner based on a parametric model of the bucket geometry, massive particle based numerical simulations and advanced optimization strategies to reduce the dimension of the design problem. The parametric model of the Pelton bucket is based on four bicubic Bézier patches and the number of free parameters is reduced to 21. The numerical simulations are performed using the finite volume particle method, which benefits from a conservative, consistent, arbitrary Lagrangian Eulerian formulation. The resulting design problem is of High-dimension with Expensive Black-box (HEB) performance function. In order to tackle the HEB problem, a preliminary exploration is performed using 2'000 sampled runners geometry provided by a Halton sequence. A cubic multivariate adaptive regression spline surro-
\end{abstract}

Christian Vessaz

EPFL, École polytechnique fédérale de Lausanne, Laboratory for Hydraulic Machines

E-mail: christian.vessaz@epfl.ch

Loïc Andolfatto

EPFL, École polytechnique fédérale de Lausanne, Laboratory for Hydraulic Machines, Avenue de Cour 33 bis, 1007 Lausanne, Switzerland

Tel.: +41 (0)21 693-2563

Fax: +41(0)21 693-3554

E-mail: loic.andolfatto@epfl.ch

François Avellan

EPFL, École polytechnique fédérale de Lausanne, Laboratory for Hydraulic Machines

E-mail: francois.avellan@epfl.ch

Christophe Tournier

LURPA, ENS Cachan, Univ. Paris-Sud, Université Paris-

Saclay, 94235 Cachan, France

E-mail: christophe.tournier@lurpa.ens-cachan.fr
Keywords Pelton turbine - Bucket shape parameterization · Design optimization · High-dimension $\cdot$ Finite volume particle method

\section{Introduction}

Over the past decades, the production of renewable energy has been constantly growing. This expansion is very likely to accelerate considering many countries reinforced their renewable energy policies. This growth includes the hydro power production at a similar pace as the other renewable energy sources.

In this context, the exploitation of hydro power potential becomes one vector of this expansion towards more renewable. The Pelton turbine is the most popular machine type for the exploitation of high head and low discharge power plants. Since the early water wheel concept featured with several double half-cylindrical buckets patented by Lester Pelton in 1880 Pelton (1880), the geometry of Pelton runners illustrated in Fig. 1 has been considerably improved. Most of the progress made take their roots in practical experience, know-how and extensive experimental tests.

In the new context where harvesting small hydro potentials can become economically viable, there is a need to provide solutions to reduce the design cycle time and the design cost for Pelton runners. Such objectives commonly relies on the use of numerical simulation tools and optimization techniques to solve the runner design problem.

The design problem addressed in this paper can be informally described as finding the runner geometry providing the best performance. The implementation of a design methodology to solve it therefore relies on 
three underlying main pillars Falcidieno et al (2014). The runner geometry can be defined by a set of design parameters instantiating a parametric model. The performance of a runners can be evaluated according to a performance indicator, generally computed through numerical simulations. Finally, finding the design parameters leading to the best runner with respect to this performance indicator relies on the implementation of an appropriate optimization strategy.

The work presented in this paper aims at highlighting and circumventing the hurdles toward the optimal design of Pelton runners thanks to numerical simulation. The literature review of Section 2 reveals the obstacles related to he high-dimension of the problem and the computational expensiveness of the numerical simulation together with the consequences on the associated optimization problem.

A geometric model is proposed and presented in Section 3. It is based on 4 bicubic Bézier patches modeling the inner surface of the bucket and on a definition of the outer surface thanks to a thickness map. A major effort is made to keep enough degrees of freedom to avoid suboptimal geometries while reducing the number of free parameters to $z_{P}=21$.

The performance of the runners is evaluated according to the simulated torque under specified operating conditions. The FVPM simulation setup providing the simulated torque is detailed in Section 4. It allows a coarse resolution for fast simulations in the exploration phase conducted in the following of the paper as well as a fine resolution well suited for the actual solving of the optimization sub-problems resulting from this exploration.

Finally, Section 5 details the initial exploration of the design space. The emphasis is put on the strategy proposed to reduce the dimension of the optimization problem. It relies on the combination of a design parameter importance ranking together with an original clustering approach. Thanks to this exploration strategy, the initial problem that would not have been achievable with finite computing resources is decomposed into 4 sub problems of lower dimension that can be realistically addressed with conventional optimization techniques. The resolution of these smaller optimization problems is not presented to avoid impeding the paper with technical aspects of low methodological value to the scientific community.

\section{Literature review}

\subsection{Geometrical modeling}

There are very few works in literature about the $3-\mathrm{D}$ modeling of Pelton turbine buckets. Generally, the models consist in a geometric parameterization based on distances and angles which correspond to hydraulic engineering parameters. However, the development of performing products relies more and more on ComputerAided Engineering (CAE) and thus on parametric models Sobieszczanski-Sobieski and Haftka (1997). In the case of products with complex shape as for the Pelton runners, Bézier, B-spline or Nurbs patches are usually implemented. The critical problem lies in the number of design parameters that must be large enough to provide sufficient shape diversity but small enough to allow an efficient exploration of the design space.

The approach proposed by Anagnostopoulos and Papantonis (2012) consists in defining a 2-D boundary curve of the bucket and a deepest point in order to build intermediate frames and consequently the lateral surface by interpolation. The main design parameters are the length and width dimensions of the 2-D boundary, the coordinates of the deepest point and its depth. The intermediate frames dimensions are defined with scaling factors, each intermediate frames introducing 3 additional variables. Once the lateral surface of the bucket is generated, the cutout is constructed at the intersection of the bucket surface with a toroidal beam modeling the water jet and defined by two design variables representing its axis location and radius. According to the authors, the construction of the entire inner surface of the bucket can be controlled by 19 geometric variables, which is quite small regarding its complexity. The drawback of this method is that the splitter tilt angle cannot be modified as well as the cutout geometry. This approach has been reused by Solemslie and Dahlhaug (2012) to build the NUBRS description of the Pelton bucket for their reference Pelton turbine design.

Michálková and Bastl (2015) propose a geometrical description based on uniform bicubic B-splines surfaces composed of $7 \times 4$ patches to model the inner surface of the bucket. The novelty consists in trying to satisfy prescribed angle distribution along the boundary curve of the bucket which is useful to control the tangential velocities at the bucket outlet. However, as the exact solution exists in very special cases, an approximate solution is proposed. The drawback of this approach is the use of $7 \times 4$ bicubic patches which are determined by $843-\mathrm{D}$ control points which potentially represents 252 parameters. Although reductions are possible, that 
still represents a significant number of parameters to carry out the geometrical optimization.

\subsection{Numerical simulation}

The deviation of a high-speed water jet by the rotating Pelton buckets is a challenging fluid mechanics problem, which involves complex geometries, moving boundaries, free surface flows and high-pressure variations. The design approach combining numerical simulation and experimental model testing, as described by Mack et al (2014), can yet not be applied in the early stages of the design process addressed in this paper. The simulation of a Pelton runner can be investigated using either Eulerian grid-based or Lagrangian particle based numerical methods.

Mack and Moser (2002) and Jošt et al (2010) highlighted the grid influence on the efficiency prediction as well as the needs of significant computing resources to compute accurately a Pelton runner using the twophase homogenous model. Xiao et al (2012) performed a Volume Of Fluid (VOF) simulation of a rotating Pelton runner. The computed efficiency is a bit lower than the experiments and the dependance on the grid resolution is also highlighted.

Marongiu et al (2010) demonstrated that particlebased methods are well suited to compute the flow in a Pelton runner. However, particle-based methods require significant computational ressources. Židonis and Aggidis (2015) present a comparison of different Eulerian and Lagrangian solvers applied to the simulations of rotating Pelton buckets.

Anagnostopoulos and Papantonis (2012) and Xiao et al (2007) proposed a fast Lagrangian computation to design Pelton runners. However, this method is only based on the inlet and outlet velocity vectors of the particles, which provides an estimation of the integrated pressure. Neither the whole pressure field nor the exact water sheet location can be accurately computed.

In 2015, Vessaz et al (2015) investigated the use of Finite Volume Particle Method (FVPM) to simulate the flow in five rotating Pelton buckets. FVPM is a particle-based solver introduced by Hietel et al (2000) in 2000. In 2009, Nestor et al (2009) extended the method to incompressible flows. This method features an Arbitrary Lagrangian-Eulerian (ALE) formulation, which means that the computing nodes can either move with the material velocity or a user-prescribed velocity. This method is able to satisfy free surface and no-slip wall boundary conditions precisely. FVPM combines attractive features of Smoothed Particle Hydrodynamics (SPH) and conventional grid-based Finite Volume
Method (FVM). It also features the ability to include additional physics, such as silt laden flow erosion Jahanbakhsh et al (2016). Therefore, it appears as an appropriate solution for numerical estimations of the performance of Pelton runners in the presented study.

\subsection{Optimization techniques}

According to the statements from subsection 2.1, the design of a Pelton runner is likely to be high-dimensional. The numerical simulation techniques only allows to have an implicit - or black-box - evaluation of the performance of a runner at a noticeable computing cost Vessaz et al (2015).

Straightforward implementations of usual optimization techniques are generally unsuccessful to solve such High-dimension Expensive Black-box (HEB) problems Shan and Wang (2010). The expensiveness of the problem can - until a certain extent - be addressed by committing computing power accordingly. In their survey, Shan and Wang (2010) stated that a key in the HEB context is to implement a strategy to tackle the high-dimensionality.

No specific physical reason came out to help decomposing the Pelton design problem into several independent sub-problems of lower dimensions. Then, the most common approach to handle the high-dimensionality is to work on the design space reduction by two means:

- reducing the dimension by removing some design parameters or by transforming a set of correlated variables into a new smaller set of uncorrelated variables with an acceptable loss on the performance prediction;

- reducing the range of some design variables to a relevant portion of the initial design space.

One popular approach to tackle high-dimensionality is to perform a Principal Components Analysis (PCA) to identify the most important combination of design parameters Shan and Wang (2010).

When such properties are not encountered, the importance of the design parameters can still be evaluated through a sensitivity analysis. Usual techniques are also limited because of the high-dimension Sudret (2008) and because of the expensiveness of the performance function Caniou (2012). In the case of HEB, one straightforward way to rank the importance of the design parameters consists in building a surrogate model $f^{*}$ of the performance function $f$ Caniou (2012) with a reduced computing cost. The surrogate model is not used to predict the performance at unexplored points but it serves to run the sensitivity analysis without increasing the overall computing budget. This approach 

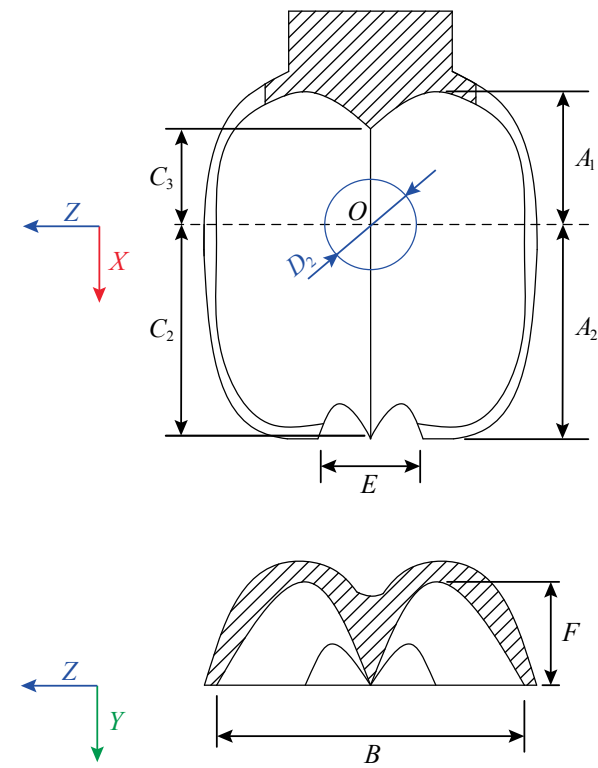

Fig. 2 General dimensions of the bucket.

proved efficient for the problem addressed in this work. It serves as a basis for the method proposed in Section 5 to define sub design spaces in which usual optimization techniques are more likely to be applied successfully.

\section{Bucket geometrical modeling}

\subsection{General approach}

The proposed parametric model of a Pelton bucket is built considering 7 parameters giving the general dimensions such as length, width and depth as represented in Fig. 2. The intersection of the water jet axis with the outer edge plane of the bucket is named $O$ and set as the origin of the bucket coordinate system. As the bucket is assumed to be symmetric with respect to the $(X, Y)$ plane, only the half bucket with positive coordinates along the $Z$ axis will be described.

Then, a set of physical points with specific properties detailed in subsection 3.2 are used to split the inner surface into four bicubic Bézier patches. These patches are defined in subsection 3.3.

The Pelton bucket performance is known to be enhanced when the outlet angles $\beta_{\overline{1}}$ decrease. This assertion is limited by the risk of heeling illustrated in Fig. 3: up to a certain angle, the water jet impinges on the outer surface of the next bucket with an associated loss of energy. To account for this heeling phenomenon, the outer surface of the bucket is defined thanks to a thickness map, as explained in subsection 3.4.

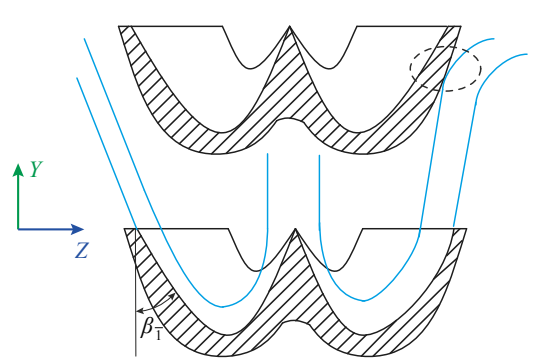

Fig. 3 Illustration of the heeling phenomenon.

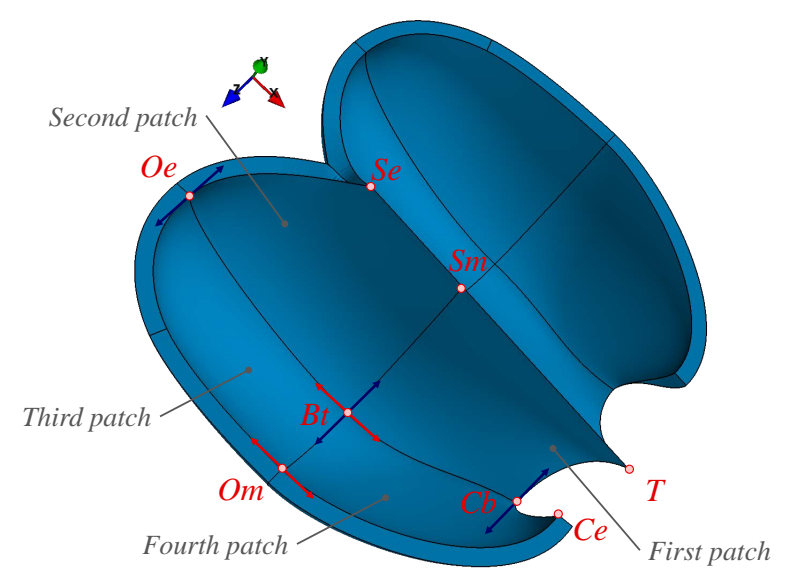

Fig. 4 Physical points considered to describe the inner surface.

\subsection{Definition of physical points}

A set of physical points with specific properties of location or tangent depicted in Fig. 4 are defined on the inner surface:

$-T$ is the tip of the bucket, located at $\left(C_{2},-y_{t}, 0\right)$;

- $C e$ is the other extremity of the cutout, with the coordinate $\left(A_{2}, 0, E / 2\right)$;

- $C b$ is the bottom point of the cutout edge, where the edge tangent is normal to the $Y$ axis, with the coordinate $\left(x_{C b},-y_{C b}, z_{C b}\right)$;

- $B t$ is the bottom of the inner surface where the surface normal is oriented along $Y$, with the coordinate $\left(x_{B t},-F, B / 4\right)$;

- $O m$ is the extreme point of the outlet edge along the $Z$ direction, with the coordinate $\left(x_{B t}, 0, B / 2\right)$;

- $S m$ is the intermediate point of the inlet edge, with the coordinate $\left(x_{B t}, y_{S m}, 0\right)$;

- $S e$ is the extreme point of the inlet edge, with the coordinate $\left(-C_{3}, y_{S e}, 0\right)$;

- Oe is the extreme point of the outlet edge along the $X$ direction, with the coordinate $\left(-A_{1}, 0, B / 4\right)$.

The inlet orientation angle $\alpha_{S m}$ is defined between the inlet edge and the $X$ direction, as depicted in Fig. 5 . 


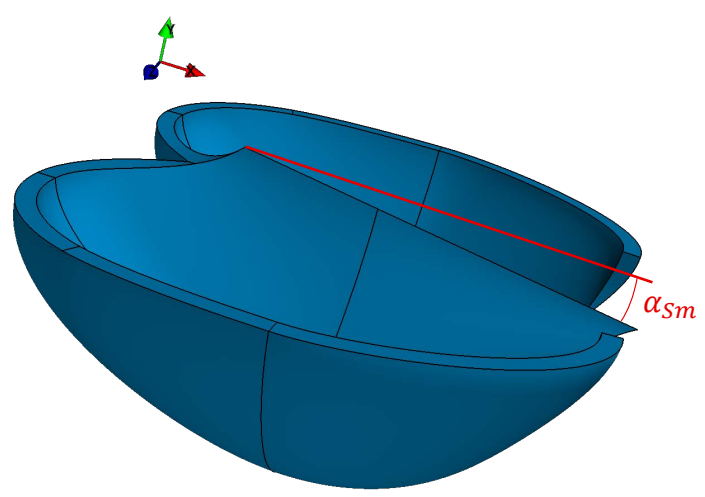

Fig. 5 Definition of the inlet orientation angle $\alpha_{S m}$.

Then the coordinates of the physical points $S m$ and $S e$ along the $Y$ direction can be expressed by eqs. (1) and (2).

$y_{S m}=C_{2} \cdot \tan \alpha_{S m}-y_{T}$

$y_{S e}=C \cdot \tan \alpha_{S m}-y_{T}$

The definition of the physical point finally requires the four additional parameters on top of the general dimensions previously defined: $\alpha_{S m}, y_{T}, x_{C b}, y_{C b}, z_{C b}$. The fifth parameter $x_{B t}$ is fixed to 0 .

\subsection{Definition of the inner surface}

Four bicubic Bézier patches are defined on the physical points. A $C^{1}$ continuity between the four surfaces is ensured by imposing a symmetry of the four control points at each vertex shared by several surfaces.

The control points of the first patch are built according to the scheme described in Fig. 6(a). The inlet angle $\beta_{1}$ defines the angle between the inlet surface and the $(X, Y)$ plane. The cutout tip angle $\alpha_{T}$ defines the orientation of the cutout edge. The cutout inlet angle $\beta_{1, C b}$ defines the angle between the cutout inlet surface and the $(Y, Z)$ plane. The cutout rotation angle $\alpha_{C b}$ defines the local rotation of the cutout edge around $Y$. The $\lambda_{k}$ are fixed ratio of the general dimensions from Fig. 2 used for the four patches.

The $C^{1}$ continuity between the first and the second patch directly defines 8 of the 16 control points of the second patch, as shown in Fig. 6(b). The other part of the inlet surface between $S m$ and $S e$ is oriented with the same $\beta_{1}$ angle with respect to the $(X, Y)$ plane. The (a)

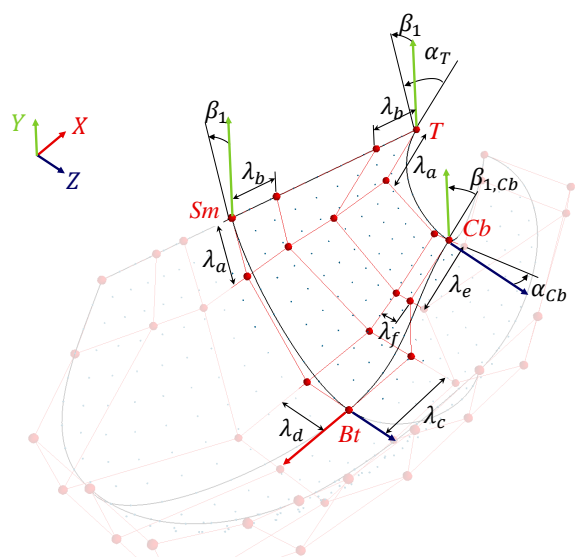

(b)

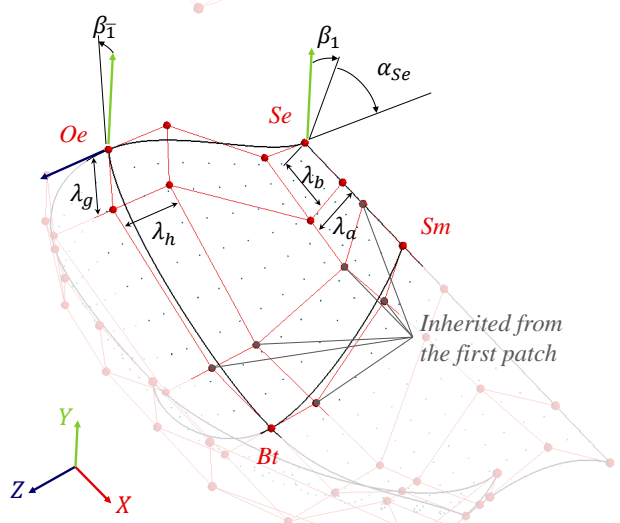

(c)

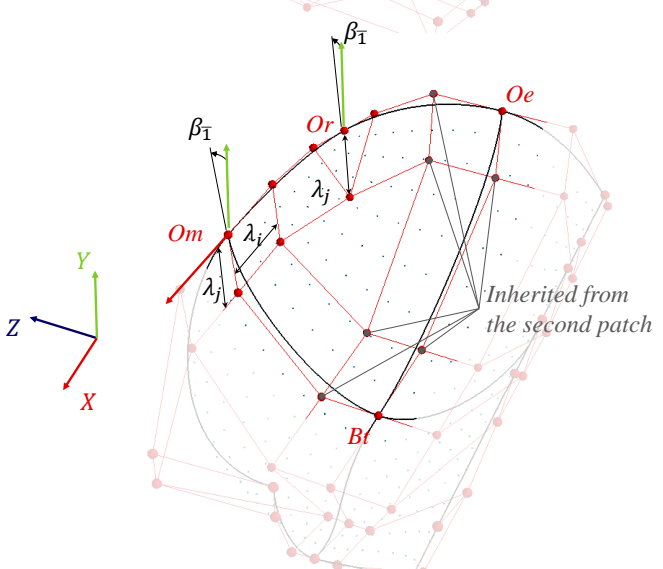

(d)

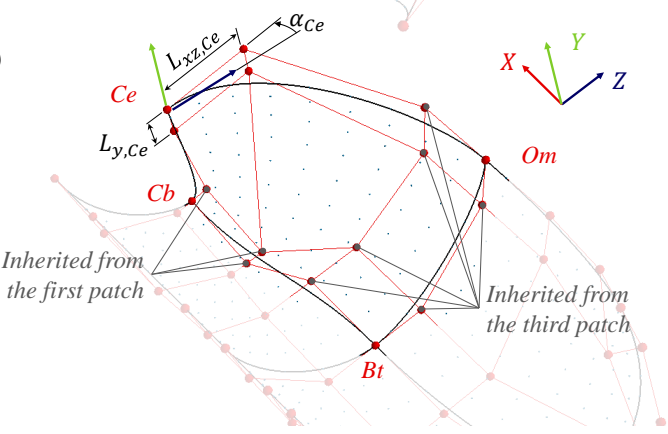

Fig. 6 Control points defining the four bicubic Bézier patches. 


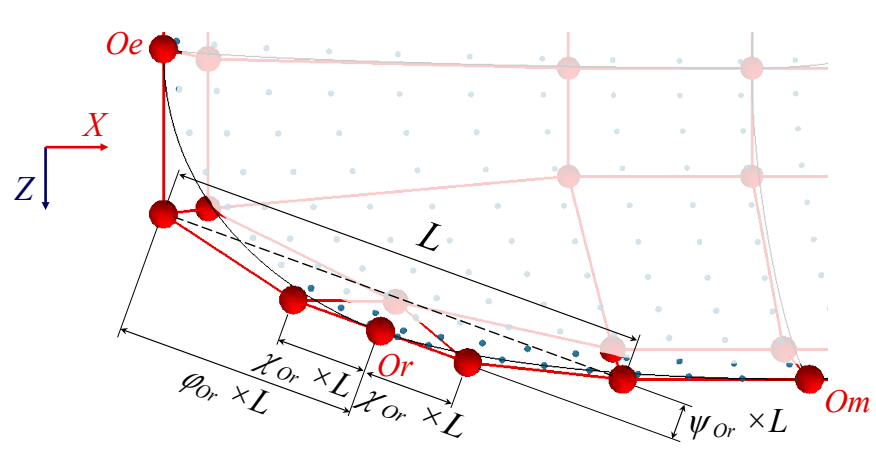

Fig. 7 Definition of the fourth vertex of the third Bézier surface with respect to physical points.

outlet surface in $O e$ is oriented with an angle $\beta_{\overline{1}}$ around $Z$. The angle $\alpha_{S e}$ is arbitrarily set to $45^{\circ}$ in order to create a smooth edge between $S e$ and $O e$. This edge is unlikely to have an influence on the hydrodynamic behavior of the bucket as it usually do not receive water Vessaz (2015).

For the third patch, 8 out of the 16 control points are derived from the second patch to ensure continuity, as depicted in Fig. 6(c). Only three of its vertex are physical points $(\mathrm{Oe}, \mathrm{Om}$ and $\mathrm{Bt}$ ). The fourth vertex named $O r$ and the associated edge orientation are built according to the definition given in Fig. 7. It requires three scalar parameters $\chi_{O r}, \phi_{O r}$ and $\psi_{O r}$ fixed to 0.75 , 0.5 and 0.075 respectively. The outlet surface is oriented with the angle $\beta_{\overline{1}}$.

Only the three control points around $C e$ remain free for the fourth patch. The other inherited control points are pictured in Fig. 6(d). The outlet edge is oriented with an angle $\alpha_{C e}$ around $Y$ in $C e$ and the cutout edge in $C e$ is oriented directly along $Y$. The position of the other control points are defined by the two distances $L_{y, C e}$ and $L_{x z, C e}$.

Finally, the inner surface requires 8 variables - or free parameters - and 13 fixed ratios of the general dimensions to be completely defined.

\subsection{Definition of the outer surface}

The outer surface is built by offsetting the patches of the inner surface along their local normal vectors with a given thickness. $P_{u v}$ represents a point of one patch of the inner surface with the parameters $(u, v)$. The normal at this point is written $\boldsymbol{n}_{u v}$. Given a thickness function $\mathcal{T}$ defined on $[0,1]^{2}$ that returns a thickness for each $(u, v)$ parameters, the point $P_{u v}^{\prime}$ of the associated patch of the outer surface is given by:

$P_{u v}^{\prime}=P_{u v}+\mathcal{T}(u, v) \cdot \boldsymbol{n}_{u v}$

For each patch of the bucket inner wall, a thickness map is defined by a set of $m \times n$ control thickness $t_{i j}$. The thickness function is of the form defined in (4) with $B_{i j}$, the Bernstein polynomials, basis functions of the Bézier patches.

$$
\mathcal{T}: \mid \begin{aligned}
{[0,1]^{2} } & \longrightarrow \mathbb{R} \\
u, v & \longmapsto \sum_{i=0}^{m} \sum_{j=0}^{n} B_{i m}(u) \cdot B_{j n}(v) \cdot t_{i j}
\end{aligned}
$$

Three values $t_{1}, t_{2}$ and $t_{3}$ are used to define the thickness maps associated to the four inner surfaces. In this study, the thickness are kept constant equals to $3 \mathrm{~mm}, 8 \mathrm{~mm}$ and $6 \mathrm{~mm}$ respectively. The sets of control thickness are graphically represented in Fig. 8. When generating the entire bucket by symmetry, the sampled points with negative coordinates along $Z$ will be removed.

\subsection{Closing the bucket volume}

The inner surfaces and outer surfaces edges are collections of isoparametric curves. A pair of curve $\mathcal{C}_{i}$ and $\mathcal{C}_{o}$ with parameter written $p$ defined on adjacent edges are considered to define the joining surface $\mathcal{S}$ between them as given in eq. (5) to yield half of a bucket.

$$
\mathcal{S}: \mid \begin{aligned}
{[0,1]^{2} } & \longrightarrow \mathbb{R} \\
p, \alpha & \longmapsto \alpha \cdot \mathcal{C}_{i}(p)+(1-\alpha) \cdot \mathcal{C}_{o}(p)
\end{aligned}
$$

A symmetry with respect to the $(X, Y)$ plane is applied to provide the entire bucket depicted in Fig. 4.

3.6 Implementation of the model for numerical simulation

The proposed model defines a Pelton bucket as a collection of parametric surfaces $\mathcal{S}(u, v)$. Despite the effort made to reduce the number of free parameters, geometrical model of a bucket depend on the 19 design parameters listed in Table 1. Locating the bucket in the runner coordinate system also requires 2 additional parameters $Y_{0}$ and $X_{0}$. Given a sampling strategy for the parameters $u$ and $v$ for each surface adapted to the simulation requirement, a discrete representation of the bucket can be generated for numerical simulations. 

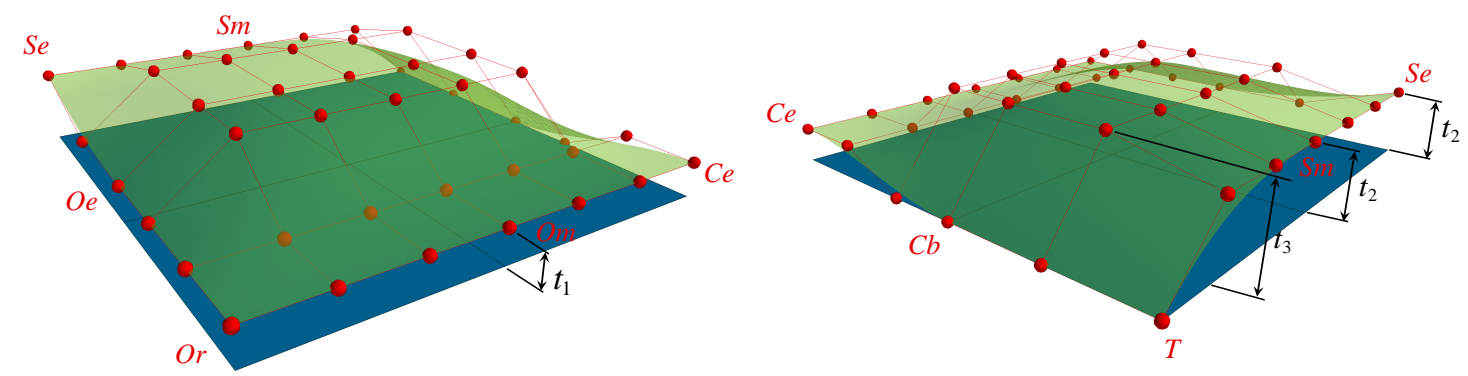

Fig. 8 Variable thickness map defining the outer surface as an offset of the inner surface.

Table 1 Inventory of the parameters describing the Pelton bucket geometry.

\begin{tabular}{ll}
\hline Category & Parameters \\
\hline General dimensions & $A_{1}, A_{2}, B, C_{2}, C_{3}, E, F$ \\
Physical points & $\alpha_{S m}, y_{T}, x_{C b}, y_{C b}, z_{C b}$ \\
Inner surface & $\alpha_{T}, \alpha_{C e}, \beta_{1}, \beta_{1, C b}, \beta_{\overline{1}}, L_{y, C e}, L_{x z, C e}$ \\
Bucket location & $Y_{0}, X_{0}$ \\
\hline
\end{tabular}

\section{Numerical simulations}

\subsection{Finite volume particle method}

In FVPM, the Sheppard interpolating or shape function $\psi$ is used to discretize the governing equations. The Sheppard function is zero-order consistent and is defined as:

$\psi_{i}(\boldsymbol{x})=\frac{W_{i}(\boldsymbol{x})}{\sigma(\boldsymbol{x})}$

where $W_{i}(\boldsymbol{x})=W_{i}\left(\boldsymbol{x}-\boldsymbol{x}_{i}, h\right)$ is the kernel function and $\sigma(\boldsymbol{x})=\sum_{j} W_{j}(\boldsymbol{x})$ is the kernel summation. The spatial resolution of the interpolation is given by the smoothing length $h$. In the present study, a rectangular top-hat kernel is used to compute the interaction vectors, which reads:

$W_{i}(\boldsymbol{x})= \begin{cases}1 & \left\|\boldsymbol{x}-\boldsymbol{x}_{i}\right\|_{\infty} \leq h, \\ 0 & \left\|\boldsymbol{x}-\boldsymbol{x}_{i}\right\|_{\infty}>h .\end{cases}$

The control volumes are replaced by particles and the exchange occurs through the interfaces defined by overlapping regions. For each pair of overlapping particles, two interaction vectors are defined. Their difference $\boldsymbol{\Delta}_{i j}$ is analogous to the area vector in FVM and is defined as:

$\boldsymbol{\Delta}_{i j}=\boldsymbol{\Gamma}_{i j}-\boldsymbol{\Gamma}_{j i}$

which depends on the interaction vector between particles $i$ and $j$ :

$\boldsymbol{\Gamma}_{i j}=\int_{\Omega} \frac{\psi_{i} \nabla W_{j}}{\sigma} d V=\int_{\Omega} \frac{W_{i} \nabla W_{j}}{\sigma^{2}} d V$.
Due to the complexity of shape functions, their integrations are usually approximated using quadrature rules over a large number of integration points. In 2011, Quinlan and Nestor (2011) developed a new FVPM in which the integrals are computed quickly and exactly for 2-D simulations. They simplified the shape functions to circular top-hat kernels and achieved a reasonable compromise between computational cost and accuracy. Recently, Jahanbakhsh et al (2014) introduced rectangular top-hat kernels to compute quickly and exactly the integrals in $3-\mathrm{D}$.

A 2-D example of particles interactions with rectangular support is given in Fig. 9(a). The top-hat kernel is less smooth than a bell-shaped kernel as shown by the contours of the Sheppard shape function given in Fig. 9(b). However, Quinlan and Nestor (2011) demonstrated that top-hat kernel allows a fast and exact computation of the interaction vector in 2-D with a circular support. In 3-D, Jahanbakhsh et al (2014) showed that the use of top-hat kernel with a rectangular support reduces significantly the cost of the integral computations in eq. (9). Therefore, the latter is simplified as:

$\boldsymbol{\Gamma}_{i j}=-\sum_{l}^{m}\left(\frac{\Delta \boldsymbol{S}_{l}}{\sigma_{l}^{+} \sigma_{l}^{-}}\right)$

where $m$ is the number of partitioned rectangles, $\Delta \boldsymbol{S}$ represents the surface vector of the partitions, $\sigma^{-}$and $\sigma^{+}$are the summation kernel inside and outside the surfaces respectively. An outline of the 2-D computation of eq. (10) is given in Fig. 9(c), where the rectangular partitions are simplified as lines segments. In this example, 4 segments are required to compute the summation of eq. (10) for particles $i$ and $j$ respectively.

The water flow is assumed inviscid and weakly compressible. The flow motion is governed by the mass and linear momentum conservation equations:

$\frac{d \rho}{d t}=-\rho \nabla \cdot \boldsymbol{C} \quad$ and $\quad \frac{d(\rho \boldsymbol{C})}{d t}=\nabla \cdot \boldsymbol{\sigma}+\rho \boldsymbol{g}$

where $\rho$ is the density, $\boldsymbol{C}$ is the velocity vector, $\boldsymbol{g}$ is the gravity vector and $\boldsymbol{\sigma}=-p \boldsymbol{I}+\boldsymbol{s}$ is the stress tensor, 


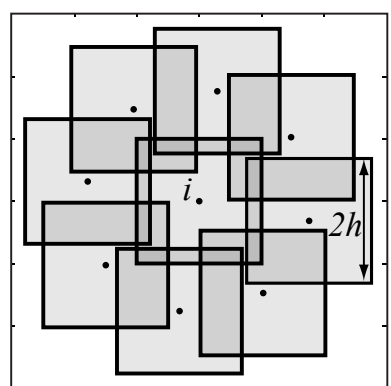

(a)

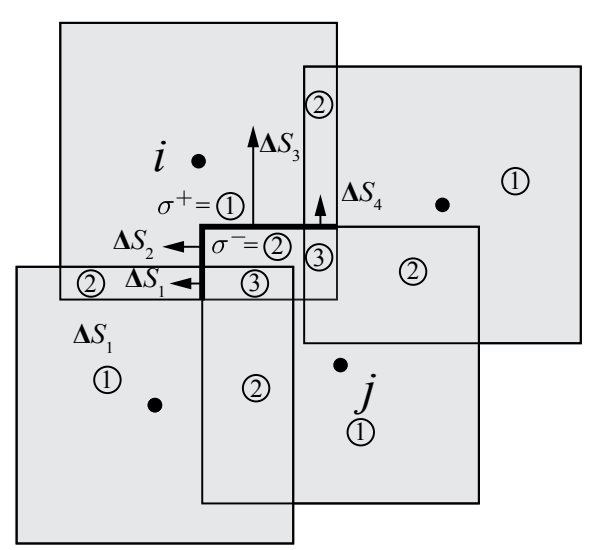

(c)

(b)

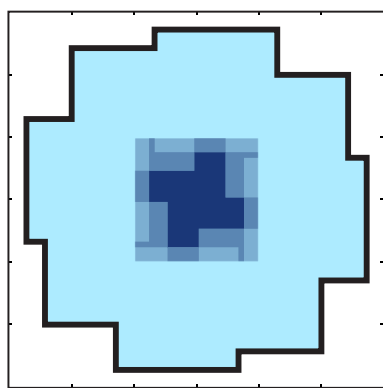

Fig. 9 Rectangular support kernels and overlapping regions (a) plotted with the contour of Sheppard shape function for the particle $i$ and top-hat kernels (b), outline of the intersection volume between particles $i$ and $j$ (c)

which includes $p$ the static pressure and $s$ the deviatoric stress contribution. In the case of an inviscid flow, the deviatoric stress contribution is equal to zero. However, in the present study, an artificial viscosity is introduced to damp the numerical oscillations Vessaz (2015). The static pressure is computed from the barotropic equation of state:

$p=\frac{\rho_{\circ} a^{2}}{7}\left(\left(\frac{\rho}{\rho_{\circ}}\right)^{7}-1\right)$

where $\rho_{\circ}$ is the reference density and $a$ is the sound speed. According to the weakly compressible assumption, the sound speed is set to $10 \cdot C_{\max }, C_{\max }$ being the discharge velocity of the water jet. This assumption is based on the weakly compressible approach of Monaghan (2005) to ensure that density variations remain below one percent and the Mach number is limited to 0.1 all along the numerical simulation, which is usual for particle-based models derived from SPH formulation. Therefore, the weakly compressible approach allows to increase the time step value compared to a pure compressible simulation by decreasing the sound speed in the CFL condition. The time integration is performed
${ }^{1.0}$ using a second-order explicit Runge-Kuta scheme and

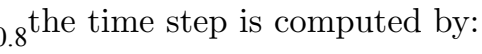

$0.6 \Delta t=0.6 \cdot \min \left(\frac{h}{a+\left\|\boldsymbol{C}_{i}\right\|}\right)$.

0.4 In order to stabilize the numerical simulations, a 0.2 correction term is applied to the mass flux following Jahanbakhsh (2014) and the $\mathrm{AUSM}^{+}$scheme of Liou (1996) is used.

${ }^{0.0}$ Moreover, a particle velocity correction is computed at each time step to ensure a uniform distribution of particles in the flow and avoid particles clustering Vessaz (2015).

To impose the solid boundary condition, one layer of wall boundary particles is located on the interface. The wall boundary particles have the property of the fluid particles, i.e. their pressure and stress are computed from governing equations of the fluid. However, their velocities are imposed equal to the wall velocity to ensure that the wall boundary particles remain attached to the solid interface. Consequently, the force applied on the boundary is given by:

$\boldsymbol{f}_{B, i}=\sum_{j \in \text { fluid }}\left(-p_{i j} \boldsymbol{I}+\boldsymbol{s}_{i j}\right) \cdot \boldsymbol{\Delta}_{i j}$.

\subsection{Input and initial setup}

In the present study, the values of the following parameters are arbitrarily set in order to have a well defined operating point for the exploration process. First, the water jet parameters are imposed as follow: the orientation of the jet is in the $-X$ direction and its inlet is located at $X=0.185 \mathrm{~m}, Y=-0.15 \mathrm{~m}$ and $Z=0.0$ $\mathrm{m}$. The discharge velocity of the water jet is $C_{\max }=$ $30.0 \mathrm{~m} \mathrm{~s}^{-1}$ with a diameter $D_{2}=0.03 \mathrm{~m}$. Second, the number of buckets of the Pelton runner is set to 20 and the rotational speed is imposed at $955 \mathrm{rpm}$.

In order to decrease the computing time, only 2 buckets are used to represent the Pelton runner. The total torque is deduced from the torque evolution in the first bucket. However, this assumption does not capture the heeling phenomenon. In this preliminary study, this phenomenon is avoided by setting a minimal $\beta_{\overline{1}}$ value, which is large enough, and checking that the healing phenomenon does not occur for the optimized geometries. In further studies, three buckets will be used and the total torque will be deduced from the second bucket. Consequently, only 0.013 seconds are simulated, which corresponds to a rotation angle of $75^{\circ}$, and is sufficient to compute the torque evolution in the first bucket. The initial setup of the simulation is presented in Fig. 10, which includes:

- the geometry of the bucket; 


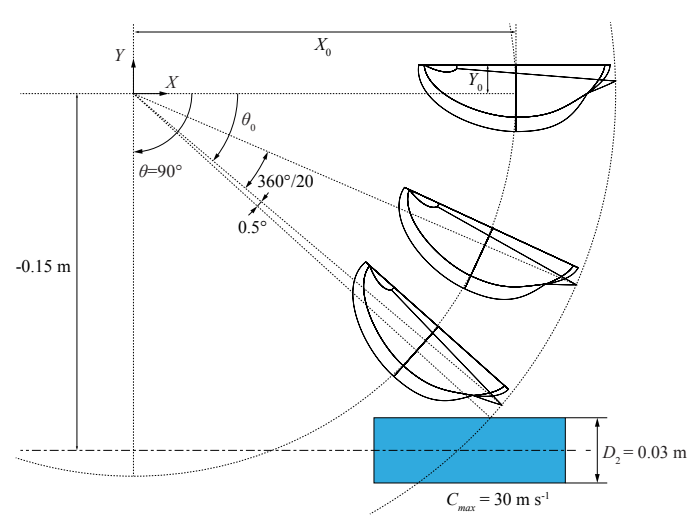

Fig. 10 Outline of the initial setup for the numerical simulations.

- two additional parameters $X_{0}$ and $Y_{0}$ which set the bucket location according to the $X$ and $Y$ Cartesian coordinate respectively;

- the initial rotation $\theta_{0}$ which is deduced from the bucket tip in order to obtain an angle of $0.5^{\circ}$ between its location and its first impact through the water jet;

- and an initially developed portion of the water jet.

The numerical simulations are performed with the FVPM solver SPHEROS developed by Jahanbakhsh et al (2012). An example of the SPHEROS results is given in Fig. 11. The particle-based representation uses the instantaneous wall pressure field to render the buckets particles. The visualization of the results is performed using the rendering software ParaView Ayachit (2015).

\subsection{Torque computation}

During the simulation, the torque is computed for each bucket and at each time step according to:

$T=\sum_{i \in \text { wall }} \boldsymbol{R}_{i} \times \boldsymbol{f}_{B, i}$

where $\boldsymbol{R}_{i}$ is the radius between the runner axis and the particle position $\boldsymbol{X}_{i}$. The evolution of the torque in each bucket as well as the total torque applying on the two buckets are given in Fig. 12 for the finest particles resolution investigated, i.e. $D_{2} / X_{\text {ref }}=50$.

In order to set the objective of the optimization process, the mean torque applied on the runner has to be evaluated from the torque evolution for bucket 1 . Therefore, the torque evolution for bucket 1 is resampled according to a given $\Delta \theta=0.025^{\circ}$ increment. Then, the torque applied on buckets 2 to 20 are deduced by shifting the torque evolution of bucket 1 . Finally, the total
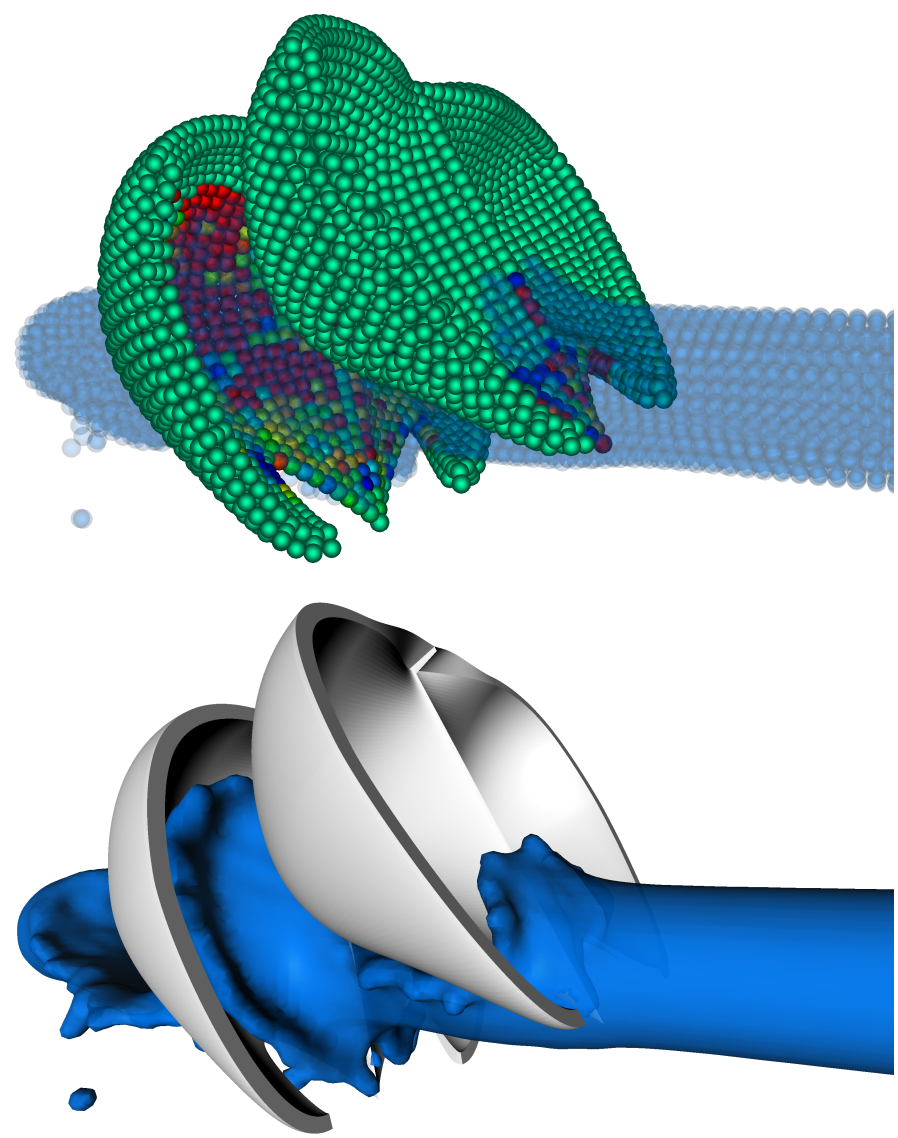

Fig. 11 FVPM simulation of two rotating buckets: particlebased representation (up) and free surface reconstruction of the water sheet (down).

torque is evaluated by summing the torque evolution of the 20 buckets. An example of the runner torque evolution is given in Fig. 13 for the particles resolution $D_{2} / X_{\text {ref }}=50$. The mean value, as well as the standard deviation, are computed to obtain global variables for the optimization process.

The convergence of the results according to the spatial discretization is shown in Fig. 14. Indeed, the FVPM ensures the convergence of the results thanks to its conservative and consistent formulation Vessaz et al (2015). This convergence is also highlighted by the mean runner torque in Table 2.

However, increasing the spatial resolution also increases drastically the computing time required for the simulations, which is highlighted in Table 2 for the five particles resolutions investigated. For the following optimization process, a coarse resolution $D_{2} / X_{\text {ref }}=10$ is selected in order to evaluate many different bucket geometries in a reasonable computing time. Choosing a consistent design space, i.e the explored design space generates buckets shaped geometries, the simulations 
Table 2 Influence of the spatial discretization on the mean runner torque, standard deviation and computing time.

\begin{tabular}{cccc}
\hline $\begin{array}{c}\boldsymbol{D}_{\mathbf{2}} / \boldsymbol{X}_{\text {ref }} \\
{[-]}\end{array}$ & $\begin{array}{c}\text { mean( } \boldsymbol{T}) \\
{[\mathrm{N}]}\end{array}$ & $\begin{array}{c}\text { std(T) } \\
{[\mathrm{N}]}\end{array}$ & $\begin{array}{c}\text { Computing time } \\
{[\text { hour }]}\end{array}$ \\
\hline 10 & 60.31 & 8.73 & 0.5 \\
20 & 64.38 & 3.44 & 4.5 \\
30 & 66.64 & 2.32 & 21.2 \\
40 & 68.32 & 2.37 & 61.2 \\
50 & 69.62 & 2.29 & 164.7 \\
\hline
\end{tabular}

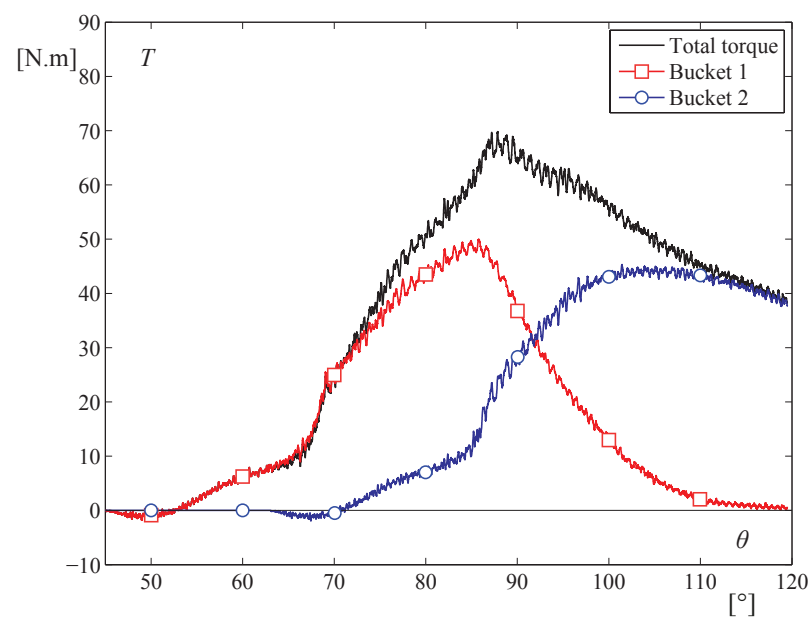

Fig. 12 Phase history of the torque for each bucket (colors) and total torque (black) for a spatial discretization of $D_{2} / X_{\text {ref }}=50$.

uncertainty is assumed to be identical for all the simulations because the physics of the flow remain the same.

The drawback of using a coarse resolution is the increase of numerical noise as highlighted by the standard deviation in Table 2. In the present paper we focus on the exploration of the design space in order to reduce the dimension of the problem. However, a finer resolution with reduced noise and uncertainty has to be used in further studies to actually solve the reduced optimization problem.

\section{Design space exploration}

\subsection{Design space definition}

The design parameters listed in Table 1 are gathered in a design parameter vector $\boldsymbol{x}$ of dimension $z_{P}=21$. The Table 3 shows the lower bound $x_{i, \min }$ and the upper bound $x_{i, \max }$ of the explored range for the associated

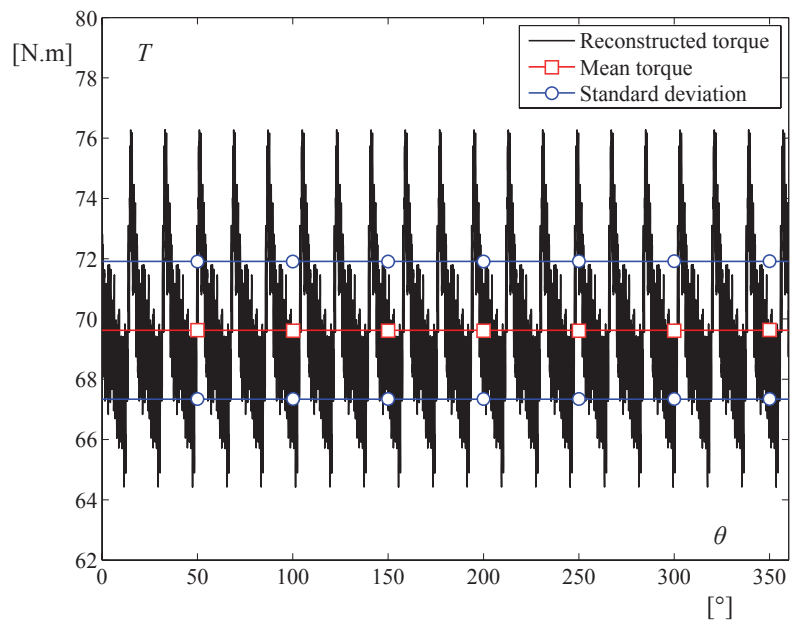

Fig. 13 Phase history of the runner torque evaluated from bucket 1 .

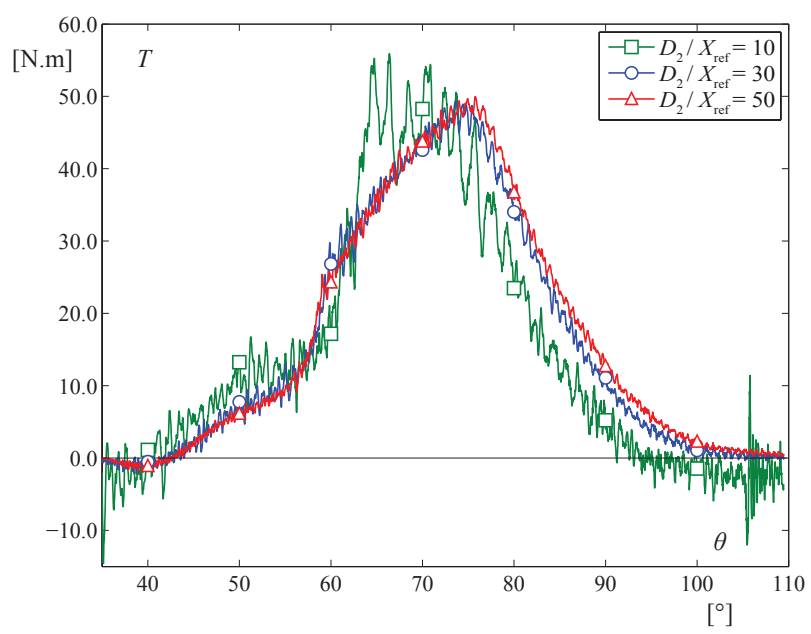

Fig. 14 Influence of the spatial discretization on the phase history of torque for bucket 1 .

geometrical parameter. The design space $\Omega$ is defined by eq. (16).

$\Omega=\prod_{i=1}^{z_{P}}\left[x_{i, \min }, x_{i, \max }\right]$

The explored range has been arbitrarily defined. This process is made easy thanks to the physical meaning associated with the parameters. Nevertheless, the consistency of this design space has been checked by sampling $N_{\text {test }}=500$ random input vectors and visualizing the associated runners without noticing any unrealistic one.

The performance $\bar{T}(\boldsymbol{x})$ representing the mean torque applied on the runner defined by the parameter vector 
Table 3 Definition of the design space $\Omega$.

\begin{tabular}{llrr}
\hline Parameters & Unit & $x_{i, \min }$ & $x_{i, \max }$ \\
\hline$A_{1}$ & {$[\mathrm{~mm}]$} & 30.0 & 60.0 \\
$A_{2}$ & {$[\mathrm{~mm}]$} & 20.0 & 60.0 \\
$B$ & {$[\mathrm{~mm}]$} & 70.0 & 110.0 \\
$C_{2}$ & {$[\mathrm{~mm}]$} & 35.0 & 60.0 \\
$C_{3}$ & {$[\mathrm{~mm}]$} & 15.0 & 45.0 \\
$E$ & {$[\mathrm{~mm}]$} & 30.0 & 50.0 \\
$F$ & {$[\mathrm{~mm}]$} & 15.0 & 35.0 \\
$y_{T}$ & {$[\mathrm{~mm}]$} & -10.0 & 5.0 \\
$x_{C b}$ & {$[\mathrm{~mm}]$} & 25.0 & 50.0 \\
$y_{C b}$ & {$[\mathrm{~mm}]$} & -20.0 & -10.0 \\
$z_{C b}$ & {$[\mathrm{~mm}]$} & 10.0 & 25.0 \\
$L_{y, C e}$ & {$[\mathrm{~mm}]$} & 2.5 & 7.5 \\
$L_{x z, C e}$ & {$[\mathrm{~mm}]$} & 12.5 & 17.5 \\
$Y_{0}$ & {$[\mathrm{~mm}]$} & 5.0 & 25.0 \\
$X_{0}$ & {$[\mathrm{~mm}]$} & 120.0 & 160.0 \\
$\alpha_{T}$ & {$[\mathrm{deg}]$} & 25.0 & 35.0 \\
$\alpha_{S m}$ & {$[\mathrm{deg}]$} & 0.0 & 15.0 \\
$\beta_{1}$ & {$[\mathrm{deg}]$} & 5.0 & 15.0 \\
$\beta_{1, C_{b}}$ & {$[\mathrm{deg}]$} & 50.0 & 70.0 \\
$\beta_{\overline{1}}$ & {$[\mathrm{deg}]$} & 2.5 & 7.5 \\
$\alpha_{C e}$ & {$[\mathrm{deg}]$} & -2.0 & 18.0 \\
\hline
\end{tabular}

$\boldsymbol{x}$ over one rotation is evaluated through FVPM simulations with the setup described in section 4.

The initial exploration of the design space was conducted with a Halton sequence containing $N_{s p}=2000$ design points. The main advantage of using a Halton sequence for the exploration lies in the ability to dynamically increase the size of the exploration sample if needed. Every new explored point of the design space will improve the uniformity of the sample Halton (1964). Iooss et al (2009) reported the efficiency of such low discrepancy sequences for the construction and the validation of surrogate models in high-dimension while pursuing similar objectives of global and uniform covering of the entire design space for exploration.

It required 50 hours of computation distributed on 20 nodes with 2 Ivy Bridge Intel Xeon E5-2650 v2 processors with 8 cores each. The average performance of the investigated population reaches 47.5 N.m. The best point provided 60.3 N.m while the worst leaded 19.6 N.m.

\subsection{Dimension reduction}

The exploration sample showed the high-non linearity of the performance function. No correlated effect of the design parameters was found either. The dimension reduction performed therefore only relies on sensitivity analysis.

A cubic Multivariate Adaptive Regression Spline model (MARS) Friedman (1991) with 108 non-constant basis functions has been built with the 2000 explored points. The MARS model has been selected for its ability to outperform other surrogate modeling techniques, such as neural networks, polynomial chaos expansion, support vector regression or Kriging, when the input dimension is higher than a dozen Andolfatto (2013). In the present case, the input dimension is 21 .

As for polynomial chaos expansion, the MARS surrogate modeling framework is also well suited to evaluate the importance of each design parameter on the performance function using only the initial exploration sample. On one hand, the model consists in a sum of piece-wise cubic spline basis functions. It is therefore possible to estimate the loss of quality of a model containing all the basis functions but the ones involving one design parameters. Friedman proposed to measure the importance of a design parameter through the difference of the Generalized Cross Validation error (GCV) between the full identified model and a model without basis functions involving this design parameter Friedman (1991). The GCV error between the mean torque $\bar{T}$ and its surrogate model $\bar{T}^{*}$ is computed according to eq. (17), where $z_{M}$ is the number of parameters of the MARS model.

$\mathrm{GCV}=\frac{1}{N_{s p}} \frac{\sum_{j=1}^{N_{s p}}\left(\bar{T}\left({ }^{j} \boldsymbol{x}\right)-\bar{T}^{*}\left({ }^{j} \boldsymbol{x}\right)\right)^{2}}{\left(1-\frac{z_{M}}{N_{s p}}\right)^{2}}$

Removing basis functions involving one design parameter decreases the number of parameters $z_{M}$ of the model but increases the difference between the actual values $\bar{T}$ and the modeled values $\bar{T}^{*}$. Therefore, a high increase of the GCV when a design parameter is removed implies a high importance of this design parameter.

On the other hand, the analytic expression of the model allows to perform directly an ANalysis Of VAriance (ANOVA). The impact of each design parameter on the performance over the design space is estimated according to the cumulative standard deviation $\sigma$ of the basis functions involving this parameter. It can be interpreted in a manner similar to a standardized regression coefficient in a linear model Friedman (1991).

With this strategy, the surrogate model is never used to predict the performance at unexplored points. The prediction error is therefore not a direct matter of concern. Yet, the quality of the surrogate model must still be assessed to ensure that all the trends of the performance function are well captured. It can be measured according to the coefficient of determination $R^{2}$ defined in eq. (18). The strategy is only applicable if 
$R^{2}$ is close enough to one.

$$
R^{2}=1-\frac{\sum_{j=1}^{N_{s p}}\left(\bar{T}\left({ }^{j} \boldsymbol{x}\right)-\bar{T}^{*}\left({ }^{j} \boldsymbol{x}\right)\right)^{2}}{\sum_{j=1}^{N_{s p}}\left(\bar{T}\left({ }^{j} \boldsymbol{x}\right)-\overline{\bar{T}\left({ }^{j} \boldsymbol{x}\right)}\right)^{2}}
$$

with:

$\overline{\bar{T}\left({ }^{j} \boldsymbol{x}\right)}=\frac{1}{N_{s p}} \sum_{j=1}^{N_{s p}} \bar{T}\left({ }^{j} \boldsymbol{x}\right)$

It the present case, the coefficient of determination $R^{2}$ reached a value of 0.9773 , which has been considered sufficient.

Table 4 presents the increases of generalized cross validation error $\triangle \mathrm{GCV}$ and the cumulative standard deviation $\sigma$ related to each design parameters. The parameters are sorted in decreasing importance and the ranks $R_{G C V}$ and $R_{\sigma}$ of each parameter are also presented in Table 4. The two importance indicators provide almost the same ranking between parameters. The five parameters which have the most influence are related to the runner diameter, cutout shape and bucket depth. The seven last parameters have a negligible influence on the performance function. The associated dimensions of the design space can therefore be left unexplored in further studies. These parameters are related to the physical point $C e$, which is not an active surface for the torque generation. The inlet and outlet angles, $\beta_{1}$ and $\beta_{\overline{1}}$, feature also a negligible influence due to the well chosen range of exploration, i.e. as small as manufacturable and to avoid the heeling phenomenon.

\subsection{Range reduction thanks to clustering}

The range reduction aims at identifying shrunk area of the design space in which solving an optimization problem will require less efforts. Many methods have been reported in the literature Shan and Wang (2010), but most of them require a dynamic sampling of the original design space. In the present case, a method based on the already explored design points is proposed to avoid supplementary computation. It consists in the identification of areas of high performance within the design space. To do so, the proposed algorithm yields clusters of runners with similar geometries and high performance. The design points in each cluster are further analyzed to define a sub design spaces of reduced dimension and reduced range in which an optimization problem can be solved.
Table 4 Relative importance of the design parameters estimated on the MARS model of the performance function, measured thanks to the Generalized Cross Validation error GCV and the cumulative standard deviation $\sigma$.

\begin{tabular}{|c|c|c|c|c|}
\hline Parameter & $\Delta \mathrm{GCV}$ & $\mathbf{R}_{\mathrm{GCV}}$ & $\sigma$ & $\mathbf{R}_{\sigma}$ \\
\hline$X_{0}$ & 332.06 & 1 & 21.87 & 1 \\
\hline$C_{2}$ & 24.26 & 2 & 5.66 & 3 \\
\hline$x_{C b}$ & 22.77 & 3 & 6.14 & 2 \\
\hline$y_{C b}$ & 20.41 & 4 & 3.48 & 8 \\
\hline$F$ & 18.20 & 5 & 4.23 & 4 \\
\hline$z_{C b}$ & 16.86 & 6 & 4.14 & 5 \\
\hline$y_{T}$ & 16.33 & 7 & 3.97 & 7 \\
\hline$E$ & 14.63 & 8 & 4.01 & 6 \\
\hline$A_{2}$ & 10.76 & 9 & 2.86 & 9 \\
\hline$B$ & 10.44 & 10 & 1.87 & 11 \\
\hline$\alpha_{S m}$ & 8.74 & 11 & 1.91 & 10 \\
\hline$Y_{0}$ & 2.80 & 12 & 0.55 & 12 \\
\hline$C_{3}$ & 1.51 & 13 & 0.29 & 13 \\
\hline$\beta_{1, C b}$ & 1.37 & 14 & 0.19 & 14 \\
\hline$A_{1}$ & 0.00 & 15 & 0.00 & 15 \\
\hline$\alpha_{C e}$ & 0.00 & 15 & 0.00 & 15 \\
\hline$\alpha_{T}$ & 0.00 & 15 & 0.00 & 15 \\
\hline$\beta_{1}$ & 0.00 & 15 & 0.00 & 15 \\
\hline$\beta_{\overline{1}}$ & 0.00 & 15 & 0.00 & 15 \\
\hline$L_{y, C e}$ & 0.00 & 15 & 0.00 & 15 \\
\hline$L_{x z, C e}$ & 0.00 & 15 & 0.00 & 15 \\
\hline
\end{tabular}

For the proposed approach, only the $N$ best runners and their associated design points $\left\{{ }^{j} \boldsymbol{x}\right\}_{j \in 1, \ldots, N}$ are considered. A distance matrix $\boldsymbol{D}$ is computed. Each term of the matrix is defined according to eq. (20).

$d_{j k}=\left\|{ }^{j} \boldsymbol{x}_{r}-{ }^{k} \boldsymbol{x}_{r}\right\|_{2}$

where the standardized design point ${ }^{j} \boldsymbol{x}_{r}$ is the image of ${ }^{j} \boldsymbol{x}$ by the linear application that maps the design space $\Omega$ onto $[0,1]^{21}$. The matrix is symmetric with zeros on the diagonal. An initial graph with $N$ nodes representing the $N$ runners is drawn without initial arc. The set of arcs in the graph is denoted $\mathcal{P}$. Then, the closest runners $j$ and $k$ satisfying eq. (21) are sequentially searched and connected in the graph until each runner has at least one arc.

$$
(j, k)=\underset{\substack{j<k \\(j, k) \notin \mathcal{P}}}{\arg \min } d_{j k}
$$

This process forms clusters of connected runners with similar geometries.

With $N=10$, the average distance between point is 1.612. The clustering graph with the smallest distance between runners is presented in Fig. 15. It yields four clusters. The associated buckets are depicted in figures 16 to 19 . 


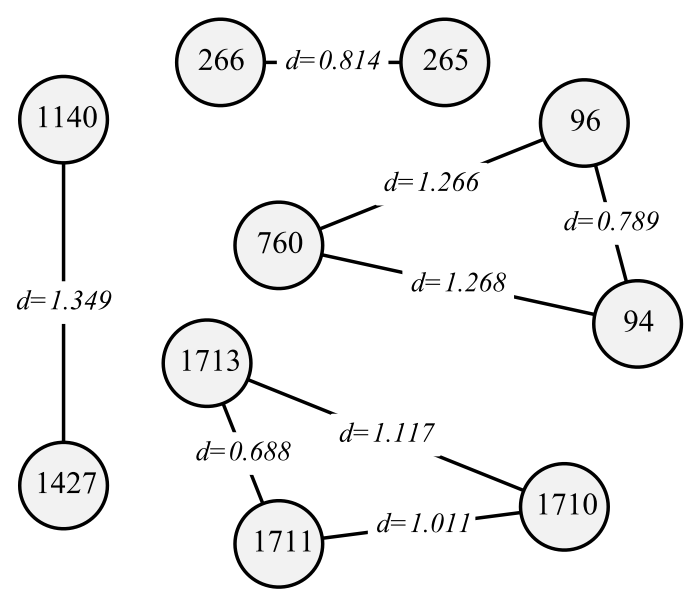

Fig. 15 Clustering graph representing the $N=10$ best runners identified by their index in the explored sample.

To define a new reduced design space associated to each cluster, the range $\Delta_{r, i}$ of the reduced coordinates $x_{r, i}$ associated with each design parameter $i$ is computed. If the range falls below an arbitrary threshold $\Delta_{\text {min }}$, meaning that the associated design parameter is almost constant within the cluster, the associated dimension no longer requires to be explored. Otherwise, the associated dimension must still be explored in the vicinity of the domain occupied by the design points of the cluster.

The design parameters with a negligible impact on the performance function identified in the previous subsection can be fixed at the mean value within the cluster.

The Table 5 shows the four sub design spaces built with $\Delta_{\min }$ fixed at $5 \%$. Four design problems of lower dimensions - from 7 to 11 instead of the initial 21 - and with smaller range can be formulated based upon these results.

\section{Conclusion}

The novel contribution of this paper lies in proposing a framework addressing the major difficulties toward the design optimization of a Pelton runner. It is chained along three main links.

First, the proposed parametric model of a Pelton bucket consists of four bicubic Bézier patches with $C^{1}$ continuity. It requires only 21 parameters while keeping enough degrees of freedom to conduct the geometrical optimization. It also features straightforward discretization capabilities to be automatically linked to the numerical simulation solver.

Then, the FVPM simulation is a state-of-the-art convenient and accurate tool to capture the deviation

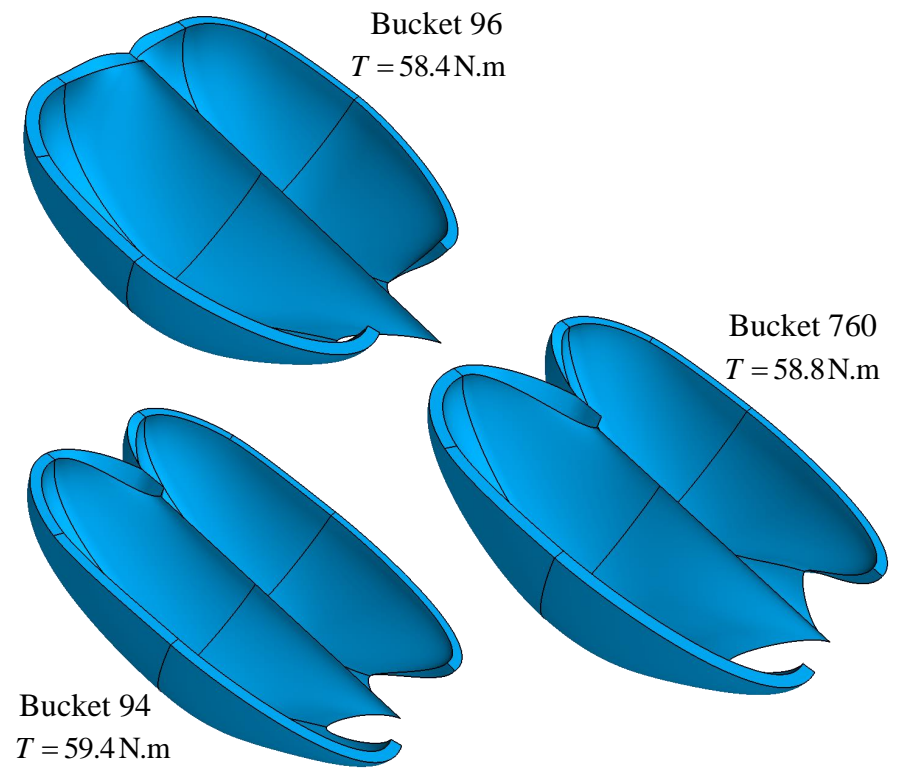

Fig. 16 Buckets from the runners of the cluster 1.

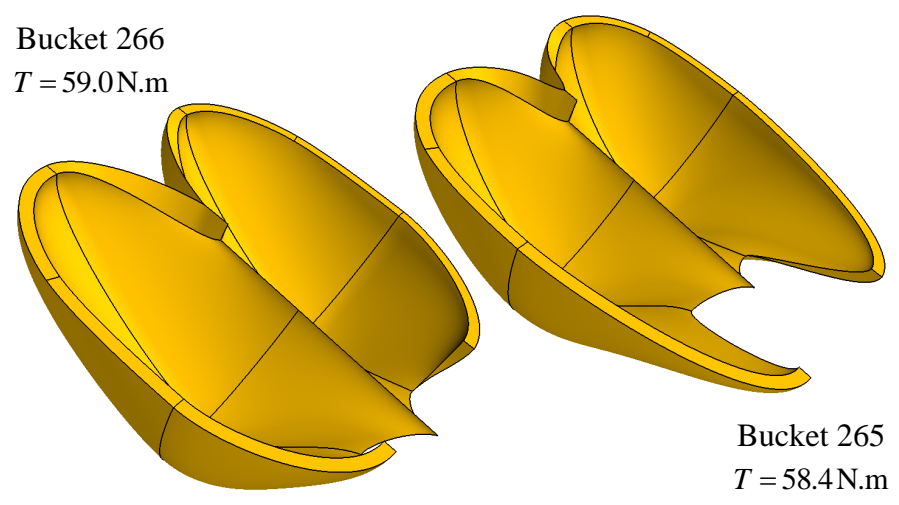

Fig. 17 Buckets from the runners of the cluster 2 .

of a water jets by rotating Pelton buckets. The total runner torque is evaluated from the torque evolution in a single bucket to reduce the computing time. However, a coarse resolution has to be selected for the numerical simulations in order to evaluate many different bucket geometries in a reasonable computing time for the exploration and dimension reduction purpose pursued in the last part of the paper. But the same simulation setup with a fine resolution can be applied for solving the reduced optimization sub-problems as in Vessaz et al (2013).

Addressing the geometrical modeling and the evaluation of the runner's performance through numerical simulation leads to a High-dimension Expensive Blackbox problem. Such problems can not be addressed directly with usual optimization technique. Therefore, the last aspect of the presented work focuses on reducing the dimension of the problem and the range of the ex- 
Table 5 Sub design spaces associated to each cluster defined by fixed value or range of each design parameter.

\begin{tabular}{|c|c|c|c|c|c|c|c|c|}
\hline \multirow[b]{2}{*}{ Parameter } & \multicolumn{2}{|c|}{ Cluster 1} & \multicolumn{2}{|c|}{ Cluster 2} & \multicolumn{2}{|c|}{ Cluster 3} & \multicolumn{2}{|c|}{ Cluster 4} \\
\hline & $\min$ & $\max$ & $\min$ & $\max$ & $\min$ & $\max$ & $\min$ & $\max$ \\
\hline$X_{0}$ & 132.1 & 137.1 & \multicolumn{2}{|c|}{146.1} & 142.6 & 145.3 & \multicolumn{2}{|c|}{138.4} \\
\hline$C_{2}$ & 52.4 & 59.6 & 36.9 & 40.5 & \multicolumn{2}{|c|}{35.9} & 49.3 & 60.0 \\
\hline$x_{C b}$ & \multicolumn{2}{|c|}{39.8} & \multicolumn{2}{|c|}{30.2} & 39.9 & 46.5 & 31.3 & 33.3 \\
\hline$y_{C b}$ & -16.8 & -14.3 & \multicolumn{2}{|c|}{-15.0} & \multicolumn{2}{|c|}{-11.5} & \multicolumn{2}{|c|}{-12.4} \\
\hline$F$ & 27.1 & 31.0 & 29.0 & 30.2 & 16.1 & 18.5 & 29.0 & 32.5 \\
\hline$z_{C b}$ & 13.2 & 20.6 & \multicolumn{2}{|c|}{13.0} & 13.4 & 18.2 & \multicolumn{2}{|c|}{22.6} \\
\hline$y_{T}$ & \multicolumn{2}{|c|}{39.8} & -9.4 & -8.6 & -9.1 & -6.9 & -8.6 & -6.3 \\
\hline$E$ & 37.0 & 41.5 & 40.1 & 41.6 & \multicolumn{2}{|c|}{47.0} & 42.5 & 47.1 \\
\hline$A_{2}$ & 43.9 & 52.8 & 31.9 & 54.1 & 32.8 & 42.6 & 34.9 & 48.2 \\
\hline$B$ & 77.4 & 93.4 & 82.9 & 90.9 & 83.1 & 94.7 & 82.4 & 106.4 \\
\hline$\alpha_{S m}$ & \multicolumn{2}{|c|}{39.8} & \multicolumn{2}{|c|}{3.0} & 4.0 & 5.4 & 0.0 & 1.6 \\
\hline$Y_{0}$ & 11.8 & 19.4 & \multicolumn{2}{|c|}{20.1} & \multicolumn{2}{|c|}{6.9} & \multicolumn{2}{|c|}{7.4} \\
\hline$C_{3}$ & 21.3 & 41.5 & 21.0 & 23.7 & 38.0 & 41.5 & \multicolumn{2}{|c|}{35.3} \\
\hline$\beta_{1, C b}$ & 50.4 & 54.0 & \multicolumn{2}{|c|}{63.5} & 55.8 & 57.9 & \multicolumn{2}{|c|}{59.0} \\
\hline$\alpha_{T}$ & \multicolumn{2}{|c|}{26.6} & \multicolumn{2}{|c|}{31.1} & \multicolumn{2}{|c|}{28.7} & \multicolumn{2}{|c|}{29.6} \\
\hline$\beta_{1}$ & \multicolumn{2}{|c|}{7.6} & & & & & & 6 \\
\hline$\beta_{\overline{1}}$ & & 5 & & & & & & 3 \\
\hline$\alpha_{C e}$ & & & & & & & & 4 \\
\hline$L_{y, C e}$ & & 1 & & & & & & 7 \\
\hline$L_{x z, C e}$ & & & & & & & & \\
\hline$A_{1}$ & & & & & & & & \\
\hline Reduced din & & & & & & & & \\
\hline
\end{tabular}

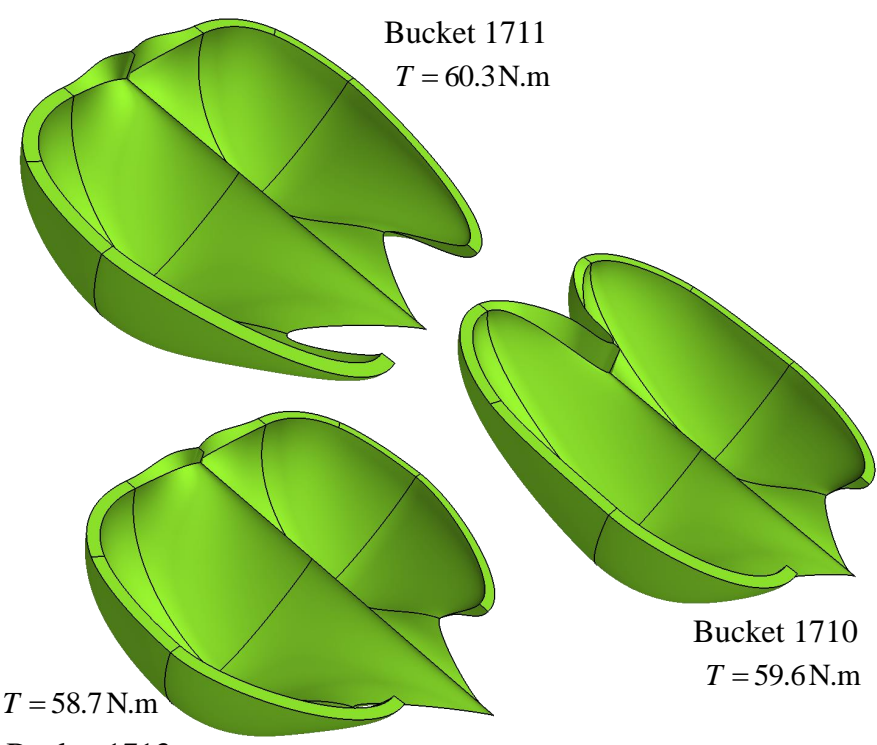

Bucket 1713

Fig. 18 Buckets from the runners of the cluster 3.

plored parameters. The initial design space is explored in order to identify the design parameters with the highest impact on the performance and the areas of the design space with the highest performance. The original clustering approach presented yields a set of optimization problems of lower dimensions with design spaces covering lower ranges that can therefore be addressed with conventional optimization techniques. This clus-

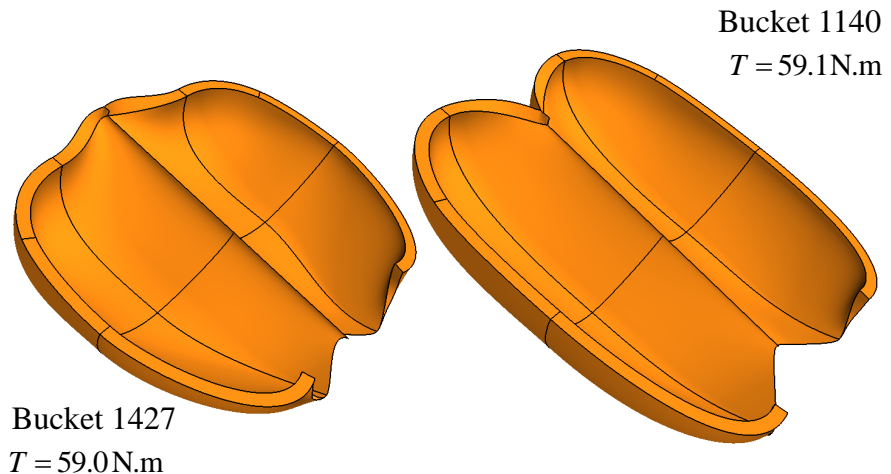

Fig. 19 Buckets from the runners of the cluster 4.

tering approach provides the last link of the proposed chain toward the design optimization of a Pelton runner. For the presented test case, four sub design spaces of dimension 7 to 11 are obtained, each of them leading to an optimization problem noticeably easier to solve than the initial one.

The next steps consists in actually solving these resulting optimization problems. As the performance of the runners in the sub design spaces are likely to vary less than in the initial space, it may be necessary to refine the particles resolution in order to increase the reliability of the simulated torque, even if it will induce an increase of the computing time. The proposed geometrical model based on an analytic description of the runner also allows the easy introduction of constraints related 
to manufacturing. Furthermore, the FVPM simulation framework is suitable for integrating other physical phenomenon such as mechanical fatigue due to cyclic loads on the buckets or erosion due to silt laden flows.

\section{References}

Anagnostopoulos JS, Papantonis DE (2012) A fast lagrangian simulation method for flow analysis and runner design in pelton turbines. Journal of Hydrodynamics, Ser B 24(6):930-941, DOI 10.1016/S1001-6058(11)60321-1

Andolfatto L (2013) Assistance à l'élaboration de gammes d'assemblage innovantes de structures composites. PhD thesis, École Normale Supérieure de Cachan, France

Caniou Y (2012) Global sensitivity analysis for nested and multiscale modelling. $\mathrm{PhD}$ thesis, Blaise Pascal University-Clermont II, France

Falcidieno B, Giannini F, Léon JC, Pernot JP (2014) Processing free form objects within a Product Development Process framework. In: Michopoulos J, Rosen D, Paredis $\mathrm{C}$, Vance $\mathrm{J}$ (eds) Advances in Computers and Information in Engineering Research, ASME, DOI 10.1115/1.860328 -ch13

Feil B, Kucherenko S, Shah N (2009) Comparison of Monte Carlo and quasi Monte Carlo sampling methods in high dimensional model representation. In: First International Conference on Advances in System Simulation, 2009. SIMUL '09., pp 12-17, DOI 10.1109/SIMUL.2009.34

Friedman JH (1991) Multivariate adaptive regression splines. The annals of statistics 19(1):1-67, URL http://www. jstor.org/stable/2241837

Halton JH (1964) Algorithm 247: Radical-inverse quasirandom point sequence. Commun ACM 7(12):701-702, DOI 10.1145/355588.365104

Hietel D, Steiner K, Struckmeier J (2000) A finite-volume particle method for compressible flows. Mathematical Models and Methods in Applied Sciences 10:1363-1382, DOI 10.1142/S0218202500000604

Iooss B, Boussouf L, Marrel A, Feuillard V (2009) Numerical study of the metamodel validation process. In: First International Conference on Advances in System Simulation, 2009. SIMUL '09., pp 100-105, DOI 10.1109/ SIMUL. 2009.8

Jahanbakhsh E (2014) Simulation of silt erosion using particle-based methods. $\mathrm{PhD}$ thesis, École polytechnique fédérale de Lausanne, no. 6284, DOI 10.5075/ epfl-thesis-6284

Jahanbakhsh E, Pacot O, Avellan F (2012) Implementation of a parallel SPH-FPM solver for fluid flows. Zetta - Numerical simulation for science and technology 1:16-20

Jahanbakhsh E, Vessaz C, Avellan F (2014) Finite volume particle method for 3-D elasto-plastic solid simulation. In: 9th SPHERIC International Workshop, Paris, France, pp 356-362

Jahanbakhsh E, Vessaz C, Maertens A, Avellan F (2016) Development of a finite volume particle method for 3-D fluid flow simulations. Computer Methods in Applied Mechanics and Engineering 298:80 - 107, DOI 10.1016/j.cma. 2015.09.013

Jošt D, Mežnar P, Lipej A (2010) Numerical prediction of a Pelton turbine efficiency. IOP Conference Series: Earth and Environmental Science 12(1), DOI 10.1088/ $1755-1315 / 12 / 1 / 012080$
Liou MS (1996) A sequel to AUSM: AUSM+. Journal of Computational Physics 129:364-382, DOI 10.1006/jcph.1996. 0256

Mack R, Moser W (2002) Numerical investigation of the flow in a Pelton turbine. In: Proceedings of the 21st IAHR Symposium on hydraulic machinery and systems, Lausanne, Switzerland, pp 373-378

Mack R, Gola B, Smertnig M, Wittwer B, Meusburger P (2014) Modernization of vertical pelton turbines with the help of cfd and model testing. In: IOP Conference Series: Earth and Environmental Science, IOP Publishing, vol 22, p 012002

Marongiu JC, Leboeuf F, Caro J, Parkinson E (2010) Free surface flows simulations in Pelton turbines using an hybrid SPH-ALE method. Journal of Hydraulic Research 48:40-49, DOI 10.1080/00221686.2010.9641244

Michálková K, Bastl B (2015) Imposing angle boundary conditions on B-spline/NURBS surfaces. Computer-Aided Design 62(0):1 - 9, DOI 10.1016/j.cad.2014.10.002

Monaghan JJ (2005) Smoothed particle hydrodynamics. Reports on progress in physics 68(8):1703-1759, DOI 10 1088/0034-4885/68/8/R01

Nestor RM, Basa M, Lastiwka M, Quinlan NJ (2009) Extension of the finite volume particle method to viscous flow. Journal of Computational Physics 228(5):17331749, DOI 10.1016/j.jcp.2008.11.003

Ayachit U (2015) The ParaView Guide: A Parallel Visualization Application, Kitware.

Pelton LA (1880) Water-wheel. URL http://www.google.com/ patents/US233692, US Patent 233692

Quinlan NJ, Nestor RM (2011) Fast exact evaluation of particle interaction vectors in the finite volume particle method. Meshfree Methods for Partial Differential Equations V pp 219-234, DOI 10.1007/978-3-642-16229-9 \_14

Shan S, Wang G (2010) Survey of modeling and optimization strategies to solve high-dimensional design problems with computationally-expensive black-box functions. Structural and Multidisciplinary Optimization 41(2):219-241, DOI 10.1007/s00158-009-0420-2

Sobieszczanski-Sobieski J, Haftka R (1997) Multidisciplinary aerospace design optimization: survey of recent developments. Structural optimization 14(1):1-23, DOI 10.1007/ BF01197554

Solemslie B, Dahlhaug O (2012) A reference Pelton turbine design. In: IOP Conference Series: Earth and Environmental Science, IOP Publishing, vol 15, p 032005, DOI $10.1088 / 1755-1315 / 15 / 3 / 032005$

Sudret B (2008) Global sensitivity analysis using polynomial chaos expansions. Reliability Engineering \& System Safety 93(7):964-979, DOI 10.1016/j.ress.2007.04.002

Vessaz C, Tournier C, Münch C, Avellan F (2013) Design optimization of a $2 \mathrm{D}$ blade by means of milling tool path. CIRP Journal of Manufacturing Science and Technology 6,157-166, DOI 10.1016/j.cirpj.2013.05.002

Vessaz C (2015) Finite particle flow simulation of free jet deviation by rotating Pelton buckets. PhD thesis, École polytechnique fédérale de Lausanne, no. 6470, DOI 10. 5075/epfl-thesis- 6470

Vessaz C, Jahanbakhsh E, Avellan F (2015) Flow simulation of jet deviation by rotating pelton buckets using finite volume particle method. Journal of Fluids Engineering 137(7):074,501-074,501, DOI 10.1115/1.4029839

Xiao YX, Han FQ, Zhou JL, Kubota T (2007) Numerical prediction of dynamic performance of Pelton turbine. Journal of Hydrodynamics, Serie B 19:356-364, DOI 10.1016/S1001-6058(07)60070-5 
Xiao YX, Cui T, Wang ZW, Yan ZG (2012) Numerical simulation of unsteady free surface flow and dynamic performance for a Pelton turbine. IOP Conference Series: Earth and Environmental Science 15(5), DOI 10.1088/ $1755-1315 / 15 / 5 / 052033$

Židonis A, Aggidis GA (2015) State of the art in numerical modelling of Pelton turbines. Renewable and Sustainable Energy Reviews 45:135-144, DOI 10.1016/j.rser.2015.01. 037 